\title{
Byurakan-IRAS Galaxy Pairs as Indicators of Starburst and Galaxy Evolution
}

\author{
A. M. Mickaelian ${ }^{1}$, L. A. Sargsyan ${ }^{1}$, and G. A. Mikayelyan ${ }^{2}$ \\ ${ }^{1}$ Byurakan Astrophysical Observatory (BAO), Armenia \\ Email: aregmick@aras.am, sarl11@yahoo.com \\ ${ }^{2}$ Department of Physics, Yerevan State University, Armenia \\ Email: gormick@mail.ru
}

Keywords. infrared: galaxies, galaxies: interactions, galaxies: starburst, galaxies: active, galaxies: evolution

The Byurakan-IRAS galaxies (BIG objects; Mickaelian 1995) are the result of a project of optical identifications of IRAS Point Source Catalog (PSC; IRAS 1988) in a 1500 square degree high-galactic latitude $\left(|b|>15^{\circ}\right)$ area based on the Digitized Sky Survey (DSS) images and the Digitized First Byurakan Survey (DFBS, or digitized Markarian survey) low-dispersion spectra. As a result, 1278 galaxies have been identified (as well as galactic objects, Byurakan-IRAS Stars [BIS]), including 42 PSC sources identified with 103 galaxies that make up 30 physical pairs and 12 multiples. Thirty-nine sources are also listed in the IRAS Faint Source Catalog (FSC; Moshir et al. 1990) allowing the use of more accurate IR positions and fluxes. These BIG objects have been observed spectroscopically with the Byurakan Observatory 2.6-m, the Special Astrophysical Observatory 6-m, and Observatoire de Haute-Provence 1.93-m telescopes and the redshifts have been measured $(0.017 \leqslant z \leqslant 0.173)$. In all cases, a physical relationship has been established, thus confirming that these tight pairs and multiples are interacting systems and/or "mergers." Using more accurate NVSS (Condon et al. 1998) and FIRST (Becker et al. 1997) radio positions, we have defined the real IR sources; it might be that either one of the components or all the components (i.e., the interaction/merging regions) are responsible for the IR radiation. It is shown that for the cases where more than one component is an IR source (30\%), the average IR luminosity is higher; typically IR luminosity greater than $10^{12} L_{\odot}$ is emitted by the whole system, which means that the interactions heat vast amounts of dust and/or trigger intense starburst processes in these objects. Hence, the maximum IR luminosity for a single spiral galaxy has been estimated as $10^{12} L \odot$. The dependence of the mean distances of components on the IR luminosity and redshift has been studied to follow the evolution of these interacting/merging systems (Mickaelian 2007). Some of the systems contain AGN (9 components are Sy2/LINER and 10 have composite spectra) that allows us investigate the interrelationship between starburst, nuclear activity, and interaction phenomena.

\section{References}

Becker, R. H., et al. 1997, The FIRST Survey Catalog, Version 2003 Apr 11, ApJ, 475, 479

Condon, J. J., et al. 1998, AJ, 115, 1693

IRAS 1988, Joint IRAS Science Working Group. IRAS Catalogs, PSC, Version 2.0, NASA

Mickaelian, A. M. 1995, Astrophysics, 38, 349

Mickaelian, A. M. 2007, Proc. IAUS235, CUP, 225

Moshir, M., et al. 1990, IRAS Catalogs, FSC, Version 2.0, NASA 